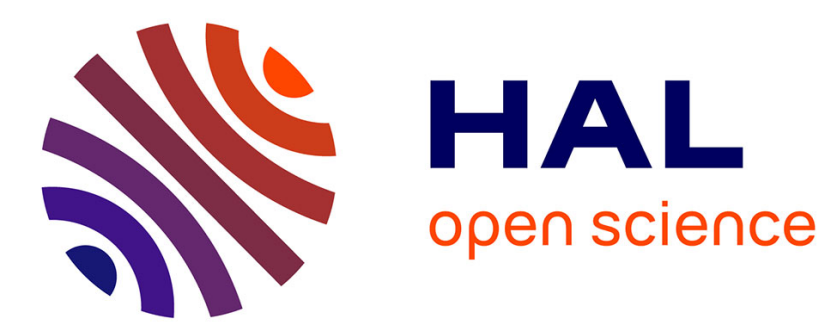

\title{
A system for real-time estimation of joint torque with evoked EMG under electrical stimulation
}

Zhan Li, Mitsuhiro Hayashibe, David Andreu, David Guiraud

\section{To cite this version:}

Zhan Li, Mitsuhiro Hayashibe, David Andreu, David Guiraud. A system for real-time estimation of joint torque with evoked EMG under electrical stimulation. ICNR: International Conference on NeuroRehabilitation, Jun 2014, Aalborg, Denmark. pp.513-520, 10.1007/978-3-319-08072-7_76 . lirmm00983717

\section{HAL Id: lirmm-00983717 https://hal-lirmm.ccsd.cnrs.fr/lirmm-00983717}

Submitted on 25 Jun 2019

HAL is a multi-disciplinary open access archive for the deposit and dissemination of scientific research documents, whether they are published or not. The documents may come from teaching and research institutions in France or abroad, or from public or private research centers.
L'archive ouverte pluridisciplinaire HAL, est destinée au dépôt et à la diffusion de documents scientifiques de niveau recherche, publiés ou non, émanant des établissements d'enseignement et de recherche français ou étrangers, des laboratoires publics ou privés. 


\title{
A System for Real-time Estimation of Joint Torque with Evoked EMG under Electrical Stimulation
}

\author{
Zhan Li, Mitsuhiro Hayashibe, David Andreu, and David Guiraud
}

\begin{abstract}
Functional electrical stimulation (FES) is a useful rehabilitation technique for restoring motor capability of spinal cord injured (SCI) patients by artificially driving muscle contractions. Real-time FES systems with online modulation ability are in great demand for clinical applications. In this work, a system for real-time estimation of joint torque with evoked electromyography (eEMG) is presented. Kalman filter (KF) is adopted and embedded into the system as the online torque estimator. The real-time estimation system would be promising toward FES control with consideration of torque changes caused by muscle fatigue.
\end{abstract}

\section{INTRODUCTION}

Functional electrical stimulation (FES) is able to restore injured subjects' motor ability by delivering electrical current pulses to their paralyzed muscles. Muscle contraction can be artificially driven through the recruitment of motor units by electrical stimulus. The elicited muscle contraction transfers muscle-tendon force to the limb segment and finally results in joint movement. The resultant joint force/torque was found to be highly correlated to evoked electromyography (eEMG) [1][2]. Explicit consideration of eEMG which represents evoked muscle electrical activities for joint torque estimation and control, could distinguish the actual moment elicited by stimulus and that by external forces. In our previous works [3][4][5], eEMG was employed for muscle fatigue tracking with surface and implanted stimulation electrodes placed on patients, and we analyzed and evaluated the estimating performance in a off-line way with the recorded data. From such off-line analysis and evaluation we see that the eEMG based estimation approach can be considered for torque prediction, implying its potential online usage for FES.

In realistic clinical environments for personalized neuroprosthetics [6][7], the FES executions divided in loops should be completed adaptively within a reasonable time duration (few ms). The closed loop modeling involving the acquired bio-feedback information (e.g., eEMG and joint torque) has to be established and identified in real-time to facilitate the stimulus modulation. In addition to evaluating and verifying the eEMG based torque estimation approaches with the recorded data in a off-line way, developing the real-time eEMG based torque estimation system is one of the key preliminary steps before implementing real-time closed loop torque control. If the real-time eEMG-based torque estimation/prediction works well, one may consider to perform real-time predictive torque control by FES.

This work is partly supported by French ANR SoHuSim Project.

The authors are with INRIA DEMAR Project and LIRMM, CNRS/University of Montpellier II, Montpellier, France. Email: $\{z l i$, hayashibe, andreu, guiraud\}elirmm.fr.
Here we list some major issues which should be considered as follows.

1) Acquisition and processing of raw eEMG and human kinetics/kinematics data should be performed synchronously to determine the true correlation between them;

2) estimation should be computed every stimulation loop;

3) stimulation sampling time should be maintained regardless of additional torque estimation process.

One key and challenging point can be that the computational identification time should be constrained to the time intervals between adjacent stimulation loop executions. Theoretically a Mwave appears once per stimulation loop post stimulus delivered to motor units, and the instant identification should be done before the next Mwave appears. Therefore, model parameters updating for every stimulation loop has to be achieved no more than the period of the loop. Otherwise, there will be largely-accumulated time lags between the real torque and estimated torque, making the estimation corrupted.

In this paper, we present a real-time eEMG based joint torque estimation system. In such system, raw eEMG (Mwave) and joint torque data are acquired synchronously and afterwards mean absolute value (MAV) of the raw eEMG is calculated per stimulation loop. Recursive Kalman filter (KF) is used as the online estimator for identifying MAV-to-torque relationship. Prediction results show promising performance of real-time eEMG based joint torque estimation system.

\section{Protocol and Data Processing}

The system tests were conducted on one able-bodied volunteer subject upon his consent at the EuroMov M2H center, Montpellier, France, as a pilot study before entering a wide protocol on spinal cord injured patients (approved by Nîmes Ethics Committee, France, 2013). The system setup for the torque estimation experiment is shown in Fig. 1. The system consists of the wireless stimulator (Vivaltis Inc., Montpellier, France) as shown in Fig. 2, eEMG (Biopac MP100, Biopac Systems Inc., Santa Barbara, CA, USA) and torque acquisition devices (Biodex 3, Shirley corp., NY, USA), and a laptop computer with the MATLAB interface for data processing and torque estimation. The subject is seated on the chair with the ankle at 90 degrees, while the joint center was aligned with the axis of a calibrated dynamometer. The shank was adjusted to be horizontal to the ground with the knee joint at 40 degrees. The foot was strapped to the pedal to transmit ankle torque to the dynamometer and to allow the optimal recording of isometric ankle torque. The bipolar AgCl EMG electrodes were positioned over the 


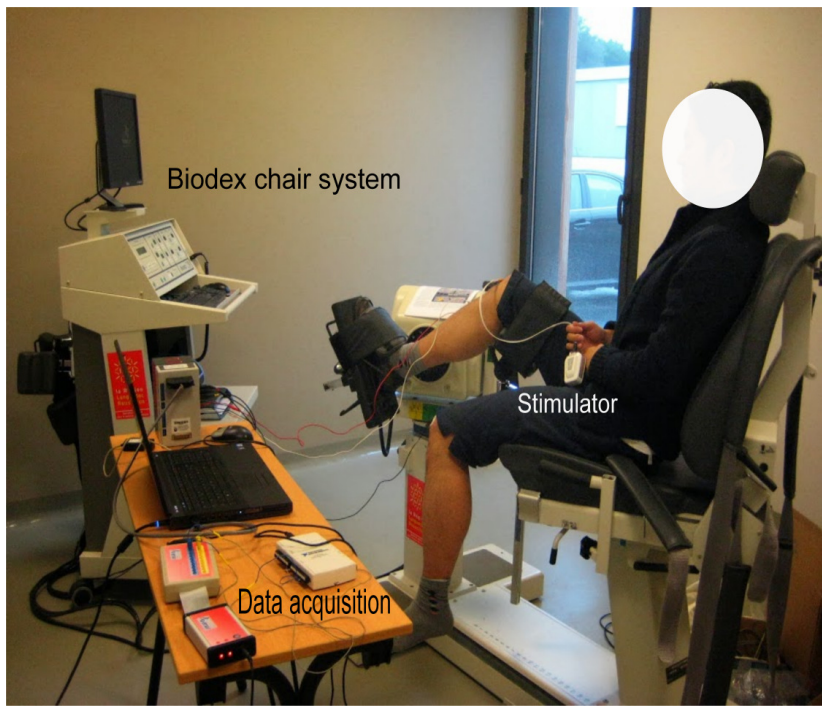

Fig. 1. Experimental setup for real-time FES-induced torque estimation

muscle belly in the direction of muscle fiber with $20 \mathrm{~mm}$ interelectrode spacing. The reference electrode was placed on the patella of another leg. Electrical current pulses generated through the wireless stimulator were delivered to the right Tibialis (TA) muscle group with surface electrodes placed. Raw eEMG of TA muscle group and ankle joint torque were recorded, amplified (gain 1000) and sampled at a frequency $f_{\text {samp }}=4096 \mathrm{~Hz}$ by an acquisition system with a 16 -bit A/D card.

The stimulation frequency was set at $f_{\text {stim }}=40 \mathrm{~Hz}$ leading to the loop execution time $25 \mathrm{~ms}$. The maximum pulse width (PW) of the stimulator was limited to $450 \mu \mathrm{s}$. The suitable stimulation intensities were found to be $25 \mathrm{~mA}$. The test session included two phases: identification and prediction phases. Each sequence contained trapezoidal trains consisting of $2 \mathrm{~s}$ stimulation $(0.5 \mathrm{~s}$ ramp-up, $1 \mathrm{~s}$ plateau and $0.5 \mathrm{~s}$ ramp-down) and $2 \mathrm{~s}$ rest. During identification phase, the plateau stimulation PW of each trapezoidal train was increased gradually with step size of from $10 \%$ to $90 \%$ of the maximum PW. After identification, the plateau stimulation PW was randomly determined within $40 \%$ to $100 \%$ of maximum $\mathrm{PW}$ in the prediction phase. The real-time $\mathrm{PW}$ series were predefined and dispatched through MATLAB Timer object and then sent to the wireless stimulation unit. The stimulation artifacts existing in the raw eEMG were removed through the following steps: 1) Calculate differences between adjacent raw eEMG samples recorded in one loop (around $f_{\text {samp }} / f_{\text {stim }} \approx 103$ samplings); 2) Compare these differences with a threshold value, if the differences are larger than it, set the corresponding adjacent raw eEMG samples as zero. During every loop the mean absolute value (MAV) of eEMG and mean value of torque were computed based on the $f_{\text {samp }} / f_{\text {stim }} \approx 103$ raw eEMG and raw torque sampling respectively. Next, when there was no stimulus, the corresponding MAV value was set to zero. Since detection of the whole area of Mwave needs exact detecting of the
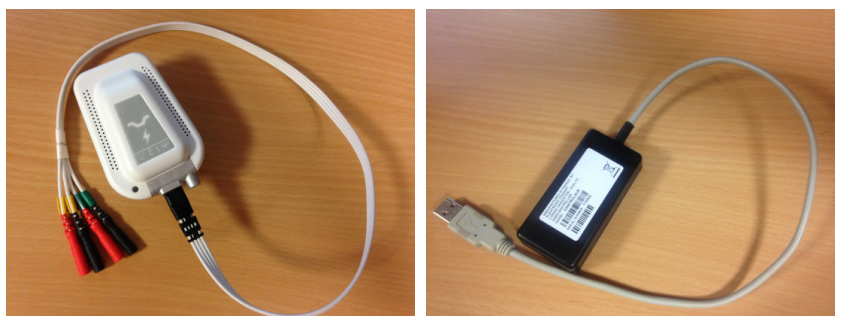

Fig. 2. Wireless stimulator (Left) with its control unit (Right)

M-wave lasting duration in real time, which could be rather difficult to detect, the MAVs of eEMG were averaged to be smoother (with a $0.8 \mathrm{~s}$ time length moving window).

\section{OnLine Kalman Filter Estimator}

In a previous work, a polynomial Hammerstein model (PHM) was used as the estimator model which could represent muscle contraction. Wwe defined contraction dynamics as the relationship between muscle activation and torque production [3][8]:

$$
y(k)=\sum_{i=1}^{l} a_{i} y(k-i)+\sum_{i=1}^{m} \sum_{j=0}^{n} b_{i} c_{j} u^{j}(k-i)+w(k)
$$

where $y(k)$ denotes model response output and $u(k)$ denotes model input, $a_{i}, b_{i}$, and $c_{j}$ denote the model parameters to be identified, $w(k)$ is the zero mean Gaussian white noise. In case of identifying relationship between stimulus and eEMG, $u(k)$ denotes normalized stimulus PW and $y(k)$ denotes MAV of eEMG; In case of identifying relationship between eEMG and torque, $u(k)$ denotes MAV of eEMG and $y(k)$ can denote analog voltage signal (with the baseline-value effect eliminated) scaling real torque.

The state space form of such PHM is described as follows:

$$
\begin{gathered}
\mathbf{x}(k)=\mathbf{A}(k) \mathbf{x}(k-1)+\mathbf{B}(k) \mathbf{\Phi}(\mathbf{u}(k-1))+\mathbf{w}(k) \\
y(k)=\mathbf{x}_{1}(k)
\end{gathered}
$$

where $\mathbf{w}(k)$ is Gaussian white noise vector and $\Phi(\mathbf{u}(k-$ $1))=\left[\begin{array}{llll}u(k-1) & u^{2}(k-1) & \cdots & u^{n}(k-1)\end{array}\right]^{T}$. Matrix $\mathbf{A}(k)$ transforms the previous state vector $\mathbf{x}(k-1)$ into the current state vector $\mathbf{x}(k)$. Coefficient $\mathbf{B}(k)$ denotes coupled linear and nonlinear parameter matrix associated with input $u(k-1)$.

Recursive KF with a forgetting factor $\lambda \in\left[\begin{array}{ll}0.9 & 1\end{array}\right]$ [3] is adopted as the online estimator in the real-time environment. Since eEMG is involved as the bio-feedback in the closed loop, dual estimators can exist. One is estimator between stimulus and eEMG, and the other is between eEMG and torque [8]. Here, the recursive KF contains two parts, first one is a priori estimate phase and the second one is a posteriori update phase.

1) a priori estimate:

$$
\begin{gathered}
\hat{\mathbf{x}}(k)=\mathbf{F}(\mathbf{x}(k-1), \mathbf{u}(k-1)) \\
\hat{\mathbf{P}}(k)=\mathbf{A}(k-1) \mathbf{P}(k-1) \mathbf{A}^{T}(k-1) / \lambda
\end{gathered}
$$



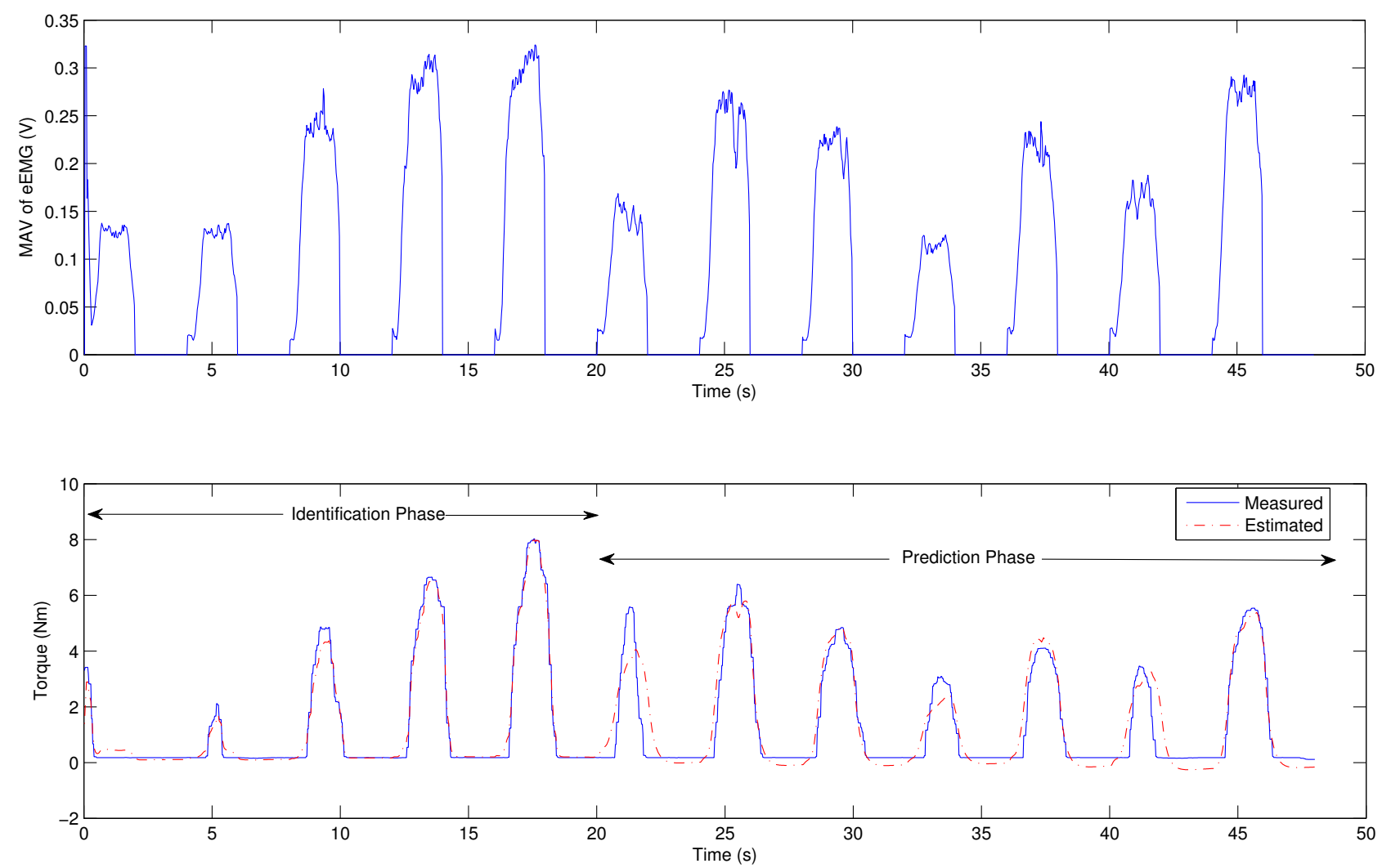

Fig. 3. Plotting of real-time online eEMG based torque estimation results. Upper: MAV of the measured eEMG; Lower: corresponding estimated and measured torque containing identification and prediction phases, from $t=20$ s identification is switched off.

$$
\hat{y}(k)=\hat{\mathbf{x}}_{1}(k)
$$

where $\hat{\mathbf{x}}(k)$ and $\hat{\mathbf{P}}(k)$ are the priori estimated state vector and plant covariance matrix respectively, and $\hat{y}(k)$ is the predicted muscle output.

2) a posteriori update:

$$
\begin{gathered}
\mathbf{S}(k)=\mathbf{H}(k) \hat{\mathbf{P}}(k) \mathbf{H}^{T}(k)+\lambda \mathbf{I} \\
\mathbf{K}(k)=\hat{\mathbf{P}}(k) \mathbf{H}^{T}(k) \mathbf{S}^{-1}(k)
\end{gathered}
$$

State vector and covariance matrix are respectively updated by

$$
\begin{gathered}
\mathbf{x}(k) \leftarrow \hat{\mathbf{x}}(k)+\mathbf{K}(k)(y(k)-\hat{y}(k)) \\
\mathbf{P}(k) \leftarrow(\mathbf{I}-\mathbf{K}(k) \mathbf{H}(k)) \hat{\mathbf{P}}(k)
\end{gathered}
$$

Such recursive KF can be implemented with the real-time measurement and acquisition system. It is worth noting that the actual obtained torque $\tau(k)=S y(k)(\mathrm{Nm})$ is scaled according to its linear relationship to the measured analog voltage $y(k)(\mathrm{V})$ exported from the analog port of the Biodex chair, where $S=43.2 / 5(\mathrm{Nm} / \mathrm{V})$ is the scaling parameter.

\section{RESUlts}

We present online identification and prediction results obtained by the established real-time system. During the whole estimation process, the predefined stimulation pattern is of trapezoidal type. Fig. 3 shows the plotting of eEMGbased torque estimation results. In the first 20 seconds the model is undergoing identification, after time instant 20s, measured torque information is not used any more and we predict the torque purely based on eEMG with the identified model.

One motivation of utilizing eEMG-based rather than PWbased estimator for torque predication is originated from the fact that eEMG-based can be of higher stability for closedloop torque control [8]. Utilization of eEMG may improve potential muscle fatigue tracking. To evaluate the prediction performance of estimation, we introduce two indexes, root mean square error (RMSE) and variance accounted for (VAF):

$$
R M S E=\sqrt{\frac{\sum_{k}^{N}\left(\tau_{m}(k)-\hat{\tau}(k)\right)^{2}}{N}}
$$

and

$$
V A F=\left(1-\frac{\operatorname{var}\left(\tau_{m}-\hat{\tau}\right)}{\operatorname{var}\left(\tau_{m}\right)}\right) \times \%
$$


TABLE I

REAL-TIME TORQUE PREDICTION PERFORMANCE EVALUATION

\begin{tabular}{|c|c|c|}
\hline Trial \# & RMSE $(\mathrm{Nm})$ & VAF $(\%)$ \\
\hline 1 & 1.09 & 89.10 \\
\hline 2 & 0.63 & 87.61 \\
\hline 3 & 0.52 & 87.82 \\
\hline 4 & 0.75 & 74.13 \\
\hline 5 & 0.66 & 67.26 \\
\hline
\end{tabular}

where $\tau_{m}$ is the actual measured torque vector and $\hat{\tau}$ is the predicted torque vector. Tab. I shows the aforementioned performance indexes for the prediction of the able-bodied subject with five trials. The overall average RMSE and VAF are respectively $0.73 \pm 0.21 \mathrm{Nm}$ and $81.14 \% \pm 9.86 \%$. The system's loop execution time of the every instant estimation was between $18 \mathrm{~ms}$ and $20 \mathrm{~ms}$ for all loops, which was not exceeding $25 \mathrm{~ms}$. The measure maximum torque of the subject was around $15 \mathrm{Nm}$, so the RMSEs of the five trials are below $1 \mathrm{Nm}$ indicating accurate predictions achieved by the system.

\section{CONClusion And Future Work}

In this work, a real-time system for online estimation of joint torque with evoked electromyography (eEMG) is presented. The whole estimation system consists of eEMG/torque acquisition and predefined-pattern stimulus delivery. Kalman filter (KF) is adopted and embedded into the system as the online estimator. Such real-time online torque estimating system produces promising prediction results. If the computational time of every stimulation loop is less than $1 / f_{\text {stim }}$, the real-time estimation system can hold the stimulation frequency $f_{\text {stim }}$ fixed. Additional functionalities of real-time FES-based torque control into the system should not much increase the executive time of each computation loop and the system operation has to be restricted within the stimulation loop duration $1 / f_{\text {stim }}$. Thus, in order to deal with this time constraint,development of efficient online computational estimation/control algorithms is important and the online torque control function is under developing based on the method as in [8]. Additionally, in order to further evaluate feasibility of the real-time torque control with eEMG as biofeedback, the next step needs to perform the experiment with SCI patients. We will demonstrate the feasibility of restoring complex movement using closed loop FES on SCI patients.

\section{REFERENCES}

[1] J. Mizrahi, M. Levy, H. Ring, E. Isakov, and A. Liberson, "EMG as an indicator of fatigue in isometrically FES-activated paralyzed muscles," IEEE Trans. Rehabil. Eng., vol. 2, no. 2, pp. 57-65, Jun. 1994.

[2] A. Erfanian, H.J. Chizeck, and R.M. Hashemi, "Using evoked EMG as a synthetic force sensor of isometric electrically stimulated muscle," IEEE Trans. Biomed. Eng., vol. 45, no. 2, pp. 188-202, Feb. 1998.

[3] Q. Zhang, M. Hayashibe, P. Fraisse, and D. Guiraud, "FES-induced torque prediction with evoked emg sensing for muscle fatigue tracking," IEEE/ASME Trans. Mech., vol. 16, no. 5, pp. 816-826, Oct. 2011.

[4] M. Hayashibe, Q. Zhang, D. Guiraud, and C. Fattal, "Evoked EMGbased torque prediction under muscle fatigue in implanted neural stimulation," J. Neural Eng., vol. 8, pp. 064001, 2011.
[5] Z. Li, M. Hayashibe, Q. Zhang, and D. Guiraud, "FES-induced muscular torque prediction with evoked EMG synthesized by NARXtype recurrent neural network," in Proc. IEEE/RSJ Int. Conf. Intell. Robots Syst., Vilamoura, Portugal, 2012, pp. 2198-2203.

[6] E. Waltz, "An electrifying awakening electrical stimulation of the spinal cord could let paralyzed people move again," IEEE Spectr., vol. 50, no. 11, pp. 46-52, Nov., 2013.

[7] D. Borton, S. Micera, J.d.R. Millán, G. Courtine, "Personalized neuroprosthetics," Sci. Transl. Med., vol. 5, no. 210, pp. 210 rv2, 2013.

[8] Q. Zhang, M. Hayashibe, and C. Azevedo-Coste, "Evoked electromyography-based closed-loop torque control in functional electrical stimulation," IEEE Trans. Biomed. Eng., vol. 60, no. 8, pp. 22992307, Aug. 2013. 